\title{
Estudo Cinético da Adsorção de Quinolina em Carvão Ativado de Casca de Dendê Funcionalizado Quimicamente
}

\author{
Nathan F. F. Potenciano, Maria E. O. Ferreira, \\ Christian G. Alonso \& Indianara C. Ostroski
}

Neste trabalho foi realizado o estudo cinético da adsorção de quinolina em sistema batelada, utilizando adsorvente funcionalizado a partir da casca do coco de dendê (CDAS). Os dados cinéticos foram obtidos para três concentrações iniciais (500, $2000 \mathrm{e}$ $5000 \mathrm{mg} \mathrm{L}-1$ ), e a concentração de quinolina remanescente na solução foi quantificada de acordo com metodologia UOP 269-10. Os dados foram ajustados aos modelos de pseudo-primeira ordem e pseudo-segunda ordem. Os resultados revelaram que a cinética de adsorção da quinolina é rápida, sendo o equilíbrio atingido após 240, 120 e 60 minutos de contato, respectivamente para as três concentrações. O modelo de pseudo-segunda ordem apresentou melhor ajuste aos dados cinéticos, revelando o caráter quimiossortivo do processo de adsorção do contaminante.

Palavras-chave: Nitrogenado Básico; Modelos Cinéticos; Adsorção Química.

In this work, it was performed the kinetic study of quinoline adsorption in bath system by using functionalized adsorbent from dendê coconut shell (CDAS). Kinetic data were obtained for three initial concentrations (500, 2000 and $5000 \mathrm{mg} \mathrm{L}-1)$, and the quinoline remaining in the solution was quantified according to UOP 269-10 methodology. The data were adjusted to the pseudo-first and pseudo-second order models. The results showed that the adsorption kinetics of quinoline is fast, with the equilibrium reached after 240, 120 and 60 minutes of contact, respectively, for the three concentrations. The pseudo-second order model presented a better fit to the kinetic data, revealing the chemisorption character of the contaminant adsorption process.

Keywords: Basic Nitrogen; Kinetic Models; Chemical Adsorption. 


\section{Introdução}

A quinolina é um dos compostos nitrogenados básicos (CNB) amplamente encontrados no refino de petróleo ${ }^{1}$. Os CNB são conhecidos por adsorver fortemente nos sítios ácidos dos catalisadores utilizados no processo de refino do petróleo, ocasionando o envenenamento dos sítios ativos. Por isto, estes compostos são considerados contaminantes do processo e devem ser removidos previamente às etapas de conversão para obtenção de derivados leves. Uma forma alternativa para remoção de compostos nitrogenados é a utilização do processo de adsorção ${ }^{2}$.

A adsorção é uma operação de transferência de massa, na qual se explora a capacidade de certos sólidos em concentrar na sua superfície determinadas substâncias (moléculas ou íons) existentes em fluidos líquidos ou gasosos, possibilitando a separação dos componentes desses fluidos ${ }^{3}$.

O carvão ativado (CA) é um adsorvente eficaz, que apresenta elevada capacidade de adsorção para vários compostos orgânicos, principalmente, porque possui uma estrutura de poros altamente desenvolvida, elevada área superficial, baixa densidade e alta resistência química. A alta capacidade de adsorção do carvão ativado também está relacionada com suas propriedades químicas. Sabe-se que a superfície do CA é essencialmente não polar, no entanto certa polaridade pode ser desenvolvida por meio de sua oxidação com ar ou com soluções de ácido nítrico, ácido sulfúrico, peróxido de hidrogênio, entre outros ${ }^{4}$. Esta prática usual visa aumentar os grupos superficiais presentes no carvão, aumentando sua especificidade e poder de adsorção.

O estudo cinético pode ser utilizado para avaliar a eficiência do processo de adsorção. O levantamento de dados cinéticos é de fundamental importância, pois permite compreender o mecanismo de adsorção, avaliar o desempenho e aplicabilidade dos adsorventes em larga escala. Sabe-se que a aplicabilidade de um adsorvente depende do seu tempo de saturação, ou seja, uma baixa velocidade de adsorção torna o processo desfavorável, uma vez que será necessário um alto tempo reacional, para adsorção eficaz de algum contaminante ${ }^{5}$.
Desta forma, o objetivo deste trabalho é avaliar a cinética de adsorção de quinolina solubilizada em tolueno, em sistema batelada, utilizando carvão ativado funcionalizado quimicamente com ácido sulfúrico (CDAS).

\section{Metodologia}

Os dados cinéticos de adsorção e o tempo de equilíbrio foram obtidos para três concentrações iniciais: 500, 2000 e $5000 \mathrm{mg} \mathrm{L}^{-1}$. Para tanto, foram utilizados frascos Erlenmeyer de $125 \mathrm{~mL}$, cada um contendo $50 \mathrm{~mL}$ de solução de concentração inicial (500, 2000 e $\left.5000 \mathrm{mg} \mathrm{L}^{-1}\right)$ de quinolina e $1 \mathrm{~g}$ do adsorvente CDAS. Os frascos foram colocados sob agitação em shaker rotativo na temperatura de $30^{\circ} \mathrm{C}$. Após tempos pré- determinados (entre 3 min e 24 h), as amostras foram retiradas e filtradas. A concentração de quinolina remanescente na fase líquida foi determinada por titulação potenciométrica, segundo método UOP 26910 para quantificação de nitrogenados básicos. Os dados cinéticos obtidos foram ajustados aos modelos de pseudoprimeira ordem e pseudo-segunda ordem.

\section{Resultados e Discussão}

A quantidade de quinolina adsorvida foi calculada pelo balanço de massa descrito pela Eq. (1)

$$
q_{t}=\frac{\left(C_{0}-C_{t}\right) V}{m}
$$

Onde, qt representa a quantidade de quinolina adsorvida na fase sólida no tempo $\mathrm{t}$ (mg g-1), C0 e Ct são as concentrações inicial e no tempo $t$ de quinolina na solução (mg L-1), respectivamente, V é o volume de solução (L), e $\mathrm{m}$ é a massa de CDAS utilizada (g). 
A Figura 1 apresenta os dados cinéticos obtidos para a adsorção da quinolina em CDAS nas diferentes concentrações testadas. A cinética do contaminante pode ser avaliada como rápida, com alta taxa de adsorção nos primeiros 25 minutos, sendo o equilíbrio atingido após 240 , 120 e 60 minutos de contato, respectivamente.

$\mathrm{Na}$ literatura constam diversos modelos para explicar a cinética de adsorção. Neste trabalho, os dados cinéticos foram ajustados aos modelos de não-linearizados de pseudoprimeira ordem e pseudo-segunda-ordem, representados nas Equações 2 e 3, respectivamente:

$$
\begin{aligned}
& q_{t}=q_{e q}\left(1-e^{-k_{1} t}\right) \\
& q_{t}=\frac{q_{e q}^{2} k_{2} t}{\left(q_{e q} k_{2} t+1\right)}
\end{aligned}
$$

em que $\mathrm{k}_{1}$ e $\mathrm{k}_{2}$ representam as constantes cinéticas de $1^{\mathrm{a}}$ ordem $\left(\mathrm{min}^{-1}\right)$ e de segunda ordem $\left(\mathrm{g} \mathrm{mg}^{-1} \mathrm{~min}^{-1}\right)$; e qeq representa a quantidade adsorvida de quinolina por grama de adsorvente no equilíbrio ( $\mathrm{mg} \mathrm{g}^{-1}$ ).

$\mathrm{O}$ ajuste aos modelos foi avaliado por meio do desvio padrão normalizado $\left(\Delta \mathrm{q}_{\mathrm{eq}}\right)$ e da determinação do coeficiente de correlação $\left(\mathrm{r}_{2}\right)$. $\mathrm{O} \Delta \mathrm{q}_{\mathrm{eq}}(\%)$ foi calculado de acordo com a Equação 4,

$$
\Delta q_{e q}(\%)=100 \sqrt{\sum \frac{\left[\frac{\left(q_{\text {eq,exp }}-q_{\text {eq, cal }}\right)^{2}}{q_{\text {eq, exp }}}\right]}{N-1}}
$$

na qual $q_{\text {eq,exp }}$ e $q_{\text {eq,cal }}$ representam a quantidade adsorvida $\left(\mathrm{mg} \mathrm{g}^{-1}\right)$ obtidas experimentalmente e pelo modelo, respectivamente; e $N$ representa o número de pontos experimentais.
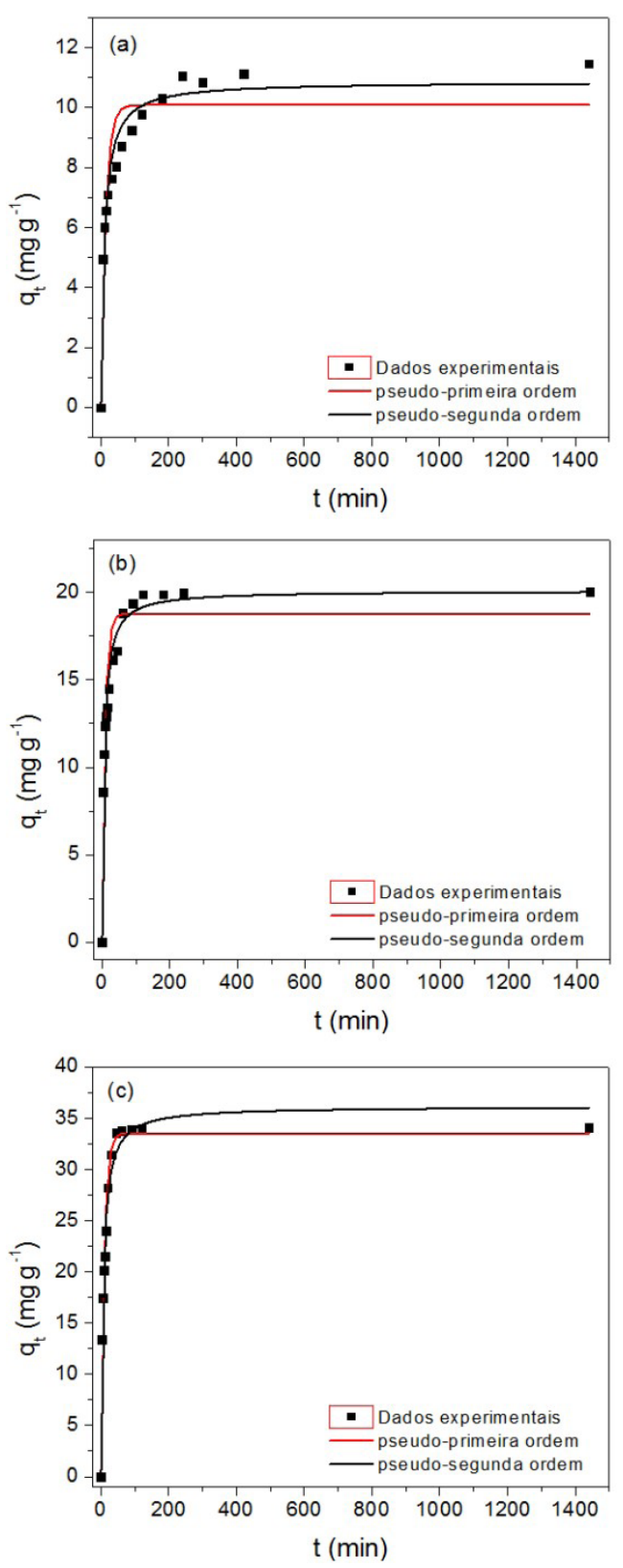

Figura 1. Cinética de adsorção da quinolina em CDAS para concentrações iniciais de (a) $500 \mathrm{mg} \mathrm{L}^{-1}$ (b) $2000 \mathrm{mg} \mathrm{L}^{-1}$ (c) $5000 \mathrm{mg} \mathrm{L}^{-1}$ e os respectivos ajustes aos modelos não linearizados de pseudo-primeira ordem e pseudosegunda ordem. 
$\mathrm{Na}$ Tabela 1 encontram-se os parâmetros cinéticos obtidos a partir das curvas ajustadas para o processo de adsorção da quinolina, bem como os valores de coeficientes de correlação $\left(\mathrm{r}^{2}\right)$ e desvios padrão normalizados $\left(\Delta_{\text {qeq }}\right)$.

A partir dos parâmetros cinéticos é possível observar que o modelo de pseudo-segunda ordem foi o que melhor se ajustou aos dados experimentais para os três sistemas, apresentando os maiores valores de $\mathrm{r}^{2}$ e menores valores de $\Delta_{\text {qeq }}$, uma vez que os resultados de qeq,exp e de qeq,cal, se aproximam. Na maioria dos casos da literatura, o modelo de pseudo-primeira ordem não se ajusta bem aos dados cinéticos e geralmente subestima os valores de qeq experimental ${ }^{6}$.

Tabela 1. Parâmetros cinéticos calculados a partir dos ajustes dos modelos não linearizados de pseudo-primeira ordem e pseudo-segunda ordem aos dados experimentais.

\begin{tabular}{|c|c|c|c|}
\hline $\begin{array}{l}\text { Concentração } \\
\text { inicial }\left(\mathrm{mg} \mathrm{L}^{-1}\right)\end{array}$ & $\mathrm{q}_{\mathrm{eq}, \exp }\left(\mathrm{mg} \mathrm{g}^{-1}\right)$ & $\begin{array}{l}\text { Pseudo- primeira } \\
\text { ordem }\end{array}$ & $\begin{array}{l}\text { Pseudo-segunda } \\
\text { ordem }\end{array}$ \\
\hline \multirow{4}{*}{500} & \multirow{4}{*}{11,46} & $q_{\text {eq, }, a l}=10,09$ & $q_{\text {eq, }, a l}=10,86$ \\
\hline & & $k_{1}=0,071$ & $k_{2}=0,010$ \\
\hline & & $r^{2}=0,8671$ & $r^{2}=0,9582$ \\
\hline & & $\Delta q_{e q}=3,20$ & $\Delta q_{e q}=1,40$ \\
\hline \multirow{4}{*}{2000} & \multirow{4}{*}{20,06} & $q_{e q, c a l}=18,77$ & $q_{\text {eq,cal }}=20,09$ \\
\hline & & $k_{1}=0,109$ & $k_{2}=0,009$ \\
\hline & & $r^{2}=0,9099$ & $r^{2}=0,9774$ \\
\hline & & $\Delta q_{e q}=1,72$ & $\Delta q_{e q}=0,04$ \\
\hline \multirow{4}{*}{5000} & \multirow{4}{*}{34,08} & $q_{\text {eq, }, c a l}=32,52$ & $q_{\text {eq, }, \text { cal }}=35,17$ \\
\hline & & $k_{1}=0,101$ & $k_{2}=0,004$ \\
\hline & & $r^{2}=0,9684$ & $r^{2}=0,9808$ \\
\hline & & $\Delta q_{e q}=1,22$ & $\Delta q_{e q}=0,85$ \\
\hline
\end{tabular}

De acordo com Ho e $\mathrm{McKay}^{7}$, o modelo de pseudo segunda-ordem descreve satisfatoriamente os processos de adsorção química que envolvem doação ou troca de elétrons entre o adsorvato e o adsorvente. Neste processo, as moléculas não são atraídas por todos os pontos da superfície do sólido, mas especificamente para os centros ativos, de maneira a formar uma monocamada, podendo haver, em seguida, a formação multicamadas por fisissorção ${ }^{8}$.

Em trabalhos publicados na literatura, observa-se que a maior parte tem como melhor ajuste dos dados o modelo de pseudo-segunda ordem, como por exemplo, pode-se citar a adsorção de quinolina em carvão ativado com $\mathrm{H}_{3} \mathrm{PO}_{4}$, utilizando dodecano como solvente'; fibra de casca de coco funcionalizada com $\mathrm{H}_{3} \mathrm{PO}_{4}$, utilizando água como solvente ${ }^{10}$; e quinolina solubilizada em meio aquoso utilizando como adsorventes as cinzas de bagaço (BFA) e carvão ativado granular (GAC) ${ }^{11}$. Neste último, foram observadas cinéticas relativamente mais lentas que a encontrada para o CDAS, utilizando a concentração de 500 $\mathrm{mg} \mathrm{L}^{-1}$, para ambos os adsorventes. O equilíbrio foi atingido após 500 minutos de tempo de contato para o GAC e após 700 minutos para o BFA. Wen et al. (2016), tal como neste trabalho, observaram altas taxas de adsorção iniciais para a quinolina. Os autores atribuem este comportamento ao fato de que a quinolina é um nitrogenado básico e contém um par de elétrons não-ligantes, o que introduz um momento dipolo significativo, resultando em forças atrativas entre o adsorvente e a molécula estudada.

\section{Conclusões}

A cinética de adsorção da quinolina em CDAS, estudada em diferentes concentrações $\left(500,2000,5000 \mathrm{mg} \mathrm{L}^{-1}\right)$, pode ser avaliada como rápida, com alta taxa de adsorção nos primeiros 25 minutos, sendo o equilíbrio atingido após 240,120 e 60 minutos de contato, respectivamente. Observou-se que o modelo de pseudo-segunda ordem apresentou resultados ligeiramente melhores com alto valor de correlação, indicando que é o modelo que melhor representa o comportamento cinético do sistema. $\mathrm{O}$ melhor ajuste a este modelo é um indicativo de que esteja ocorrendo adsorção química, envolvendo a doação ou troca de elétrons entre o adsorvato e o adsorvente.

Assim, os dados cinéticos obtidos para o sistema indicam o uso do adsorvente CDAS como promissor na remoção do contaminante nitrogenado presente em diferentes correntes de petróleo.

\section{Agradecimentos}

Os autores são gratos a CAPES pelo auxílio financeiro. 


\section{Referências Bibliográficas}

1. Ahmed, M. J. K.; Ahmaruzzaman, M Journal of Environmental Science and Technology, v. 13, n. 4, p. 1177-1188, 2016.

2. Ahmed, M. J. K.; Ahmaruzzaman, M Ind. Eng. Chem. Res., v. 48, p. 951-960, 2009.

3. Ruthven, D. M. New York: John Wiley \& Sons, 1984.

4. Rodríguez-Reinoso, F.; Molina-Sabio, M. Advances in Colloid and Interface Science, v. 76-77, p. 271-294, 1998.

5. Rodrigues, A. P. H. Dissertação (Mestrado em Engenharia de Materiais) - Centro Federal de Educação Tecnológica de Minas Gerais. Belo Horizonte - MG, 130 f. 2014.

6. Vijayaraghavan, K.; Jegan, J.; Palanivelu, K.; Velan, M. Journal of Hazardous Materials, B113: 223, 2004

7. Ho. Y. S.; Mckay, G. Water Research, v. 33, n. 2, p. 578-584, 1999.

8. Coulson, J. M.; Richardson, J. F. Lisboa: Fundação Calouste Gulbenkian, 1982. 746 p.

9. Wen, J.; Han, X.; Lin, H.; Zheng, Y.; Chu, W. Chemical Engineering Journal, v. 164, n. 1, p. 29-36, 2010.

10. Ahmed, M. J. K.; Ahmaruzzaman, M. International Journal of Environmental Science and Technology, v. 13, n. 4, p. 1177-1188, 2016.

11. Rameshraja, D.; Srivastava, V. C.; Kushwaha, J. P.; Mall, I. D. Chemical Engineering Journal, v. 181-182, p. 343-351, 2012.

\section{Nathan F. F. Potenciano, Maria E. O. Ferreira, Christian G. Alonso \& Indianara C. Ostroski}

Instituto de Química, Universidade Federal de Goiás, CEP 74690-900 Goiânia, Goiás, Brasil

*E-mail: indianara_ostroski@ufg.br 